Anaesthesist 2011 · 60:885-886 DOI 10.1007/s00101-011-1950-8

Online publiziert: 19. Oktober 2011

(c) Springer-Verlag 2011

\author{
M. Krebs $\cdot$ S. Weber-Carstens \\ Klinik für Anästhesiologie mit Schwerpunkt operative Intensivmedizin \\ und OP-Management, Charité-Universitätsmedizin Berlin, Campus \\ Virchow-Klinikum \& Charité Campus Mitte, Berlin
}

\title{
Erworbene neuromuskuläre Schwäche
}

\section{Unterschätztes Problem in der Intensivmedizin}

Im intensivmedizinischen Kontext erworbene neurologische Organfunktionsstörungen stellen eine relevante Komorbidität kritisch kranker Patienten dar. Gemäß einer aktuellen Untersuchung lag bei jedem dritten Patienten mit schwerer Sepsis eine Organfunktionsstörung des Nervensystems vor [1]. Dennoch müssen wir davon ausgehen, dass die Inzidenz der neurologisch begründeten Organkomplikationen in solchen Erhebungen erheblich unterschätzt wird.

Der Grund dafür findet sich in einer nichtverlässlichen Erfassung der neurologischen Organfunktion innerhalb weltweit etablierter Scoring-Systeme zur Messung von Organdysfunktionen auf der Intensivstation [Sequential Organ Failure Assessment Score (SOFA), Logistic Organ Dysfunction System (LODS), Multiple Organ Dysfunction Score (MODS)], in deren Rahmen die Beurteilung des Zentralnervensystems mithilfe der Glasgow Koma-Skala durchgeführt wird. Diese Skala wurde ursprünglich für die Klassifizierung und Verlaufsbeurteilung akut aufgetretener Bewusstseinsstörungen entwickelt, z. B. nach Schädel-Hirn-Trauma, Intoxikation oder akuter metabolischer Entgleisung [2], aber nicht für die Erfassung der im intensivmedizinischen Kontext erworbenen Organdysfunktion des Zentralnervensystems.

Geeignete Monitoring- und ScoringVerfahren $[3,4]$ für die zerebral begründeten neurologischen Organfunktionsstörungen, die klinisch als schweres hypo- oder hyperaktives Delir, später als ko- gnitive Funktionsstörung sichtbar werden, belegen eindrücklich, dass sie mit einer Inzidenz von $40-80 \%$ [4] sehr viel häufiger im Verlauf einer kritischen Erkrankung auftreten, als dies die Beurteilung mithilfe der Glasgow Koma-Skala nahelegt.

Die im Verlauf einer kritischen Erkrankung erworbene neuromuskuläre Organdysfunktion wird mit den zur Verfügung stehenden Scoring-Systemen zur Einschätzung der Erkrankungsschwere überhaupt nicht erfasst und stellt somit ein erhebliches Defizit in der Einschätzung der klinischen Situation der Intensivpatienten dar. Eine neuromuskuläre Organdysfunktion oder auch „intensive care unit acquired weakness" (ICUAW) wird oft erst nach erfolgreicher Behandlung der Grunderkrankung als Problem sichtbar und findet erst dann als klinische Diagnose durch z. B. physiotherapeutische Maßnahmen Aufmerksamkeit und Beachtung in der Behandlung.

Für den Patienten bedeutet die Muskelschwäche einen längeren Aufenthalt auf der Intensivstation, weil z. B. die Entwöhnung vom Respirator aufgrund muskulärer Schwäche nicht gelingt, und auBerdem eine deutlich verlängerte Rehabilitation. Dies stellt nicht nur einen erheblichen Kostenfaktor dar, sondern ist für die Patienten auch mit einer starken Einschränkung der Lebensqualität verbunden. Wie erst kürzlich in einer Fünfjahres-Follow-up-Untersuchung gezeigt werden konnte, erholen sich Patienten nach Lungenversagen (,acute respiratory distress syndrome“, ARDS) innerhalb der erste 2 bis 3 Jahre nach Entlassung von der Intensivstation (etwa $70 \%$ der erwarteten Gehstrecke werden zu diesem Zeitpunkt im 6-Minuten-Gehtest erreicht), danach stagniert der Zuwachs an Mobilität bzw. Selbstständigkeit [5].

Auch wenn die erworbene Muskelschwäche ein nichtunbekanntes Problem darstellt, wurde die ICUAW erstmals 2009 an einem "round table“ systematisiert und dieses in der Spätphase der Erkrankung heterogene Krankheitsbild in seinen Facetten definiert. In der sehr lesenswerten Übersicht „Erworbene Muskelschwäche beim kritisch Kranken" in dieser Ausgabe von Der Anaesthesist zeigen Judemann et al. eindrücklich auf, dass die im Rahmen einer kritischen Erkrankung erworbene neuromuskuläre Organdysfunktion einen signifikanten Einfluss auf den intensivmedizinischen Verlauf und die Rehabilitation der körperlichen Leistungsfähigkeit der Patienten hat.

Die Pathomechanismen, die zur „critical illness polyneuropathy" (CIP) und "critical illness myopathy" (CIM) führen, sind bisher nicht genau bekannt. Allerdings konnten Risikofaktoren bzw. Konstellationen identifiziert werden, die für deren Entstehung prädisponieren. Ist die Muskelschwäche erst einmal eingetreten, ist eine sichere Diagnostik bzw. ein Ausschluss einer anderen zugrunde liegenden Erkrankung wichtig, um die Patienten nicht vorschnell in eine „Diagnoseschublade" zu stecken und dort zu belassen. Aufgrund der limitierten Therapie- 
möglichkeiten stellt die frühe Identifikation von Patienten, die ein erhöhtes Risiko aufweisen, eine ICUAW zu entwickeln, noch bevor sich klinisch eine Muskelschwäche zeigt, eine Herausforderung und Notwendigkeit gleichermaßen dar. Da es bisher kein allgemein anerkanntes Verfahren zur Diagnostik von ICUAW gibt, wird diese regional und international unterschiedlich durchgeführt. Die Autoren setzen in der Diagnostik u. a. die Messung der Muskelmembranerregbarkeit nach direkter Muskelstimulation ein, die eine gute Methode darstellt, die Patienten, die eine Muskelschwäche entwickeln, früh im Verlauf der kritischen Erkrankung zu identifizieren [6]. Da die Therapieoptionen bei diagnostizierter ICUAW derzeit noch limitiert sind bzw. sich noch im experimentellen Stadium befinden, scheint der Schlüssel in der Verhinderung der Entwicklung einer ICUAW zu liegen; deshalb kommt der frühen Diagnose eine besondere Bedeutung zu.

Eine aufsehenerregende Arbeit [7] zeigte 2009, dass die mit einer Sedierungspause durchgeführte frühe Mobilisierung, auch von beatmeten Intensivpatienten, einen positiven Einfluss auf das funktionale Outcome der Patienten hat. Die frühe Mobilisierung der Intensivpatienten, also das Konzept der „walking ICU“, stellt die ideale Situation für Patienten auf Intensivstationen dar, auch wenn man einschränkend feststellen muss, dass eine frühe Mobilisierung nicht mit jedem Patienten in vollem Umfang möglich ist, denkt man nur an schwer Schädel-Hirn-traumatisierte Patienten oder Patienten in septischem oder kardiogenem Schock. Zudem ist es im klinischen Alltag auf einer Intensivstation bislang noch nicht möglich, mit Patienten regelhaft Gehtraining über den Flur oder in den Innenhof einer Klinik zu unternehmen, da hierfür derzeit die personellen und häufig auch die materiellen Ressourcen fehlen. Dies ist ein Thema, dem in Zukunft mehr Aufmerksamkeit geschenkt werden muss.

Judemann et al. haben in einer gelungenen Übersichtsarbeit den bisherigen wissenschaftlichen Stand anschaulich aufgearbeitet und die wichtigen Punkte der frühen Diagnostik sowie der Prävention der Entwicklung durch frühe Muskelaktivierung und Physiotherapie treffend herausgearbeitet. Auch wenn die therapeutischen Möglichkeiten noch begrenzt sind, besteht doch Hoffnung, in Zukunft weitere Instrumente an die Hand zu bekommen, um die frühe Mobilisierung von Intensivpatienten zu realisieren und damit CIP und CIM in der Entstehung wirksam präventiv entgegenzutreten.
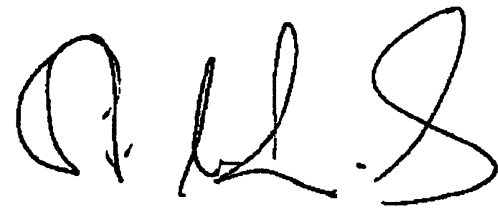

Steffen Weber-Carstens

\section{Korrespondenzadresse}

\section{Dr. S. Weber-Carstens}

Klinik für Anästhesiologie mit

Schwerpunkt operative

Intensivmedizin und OP-Management,

Charité-Universitätsmedizin Berlin, Campus

Virchow-Klinikum \& Charité Campus Mitte

Augustenburger Platz 1, 13353 Berlin

steffen.weber-carstens@charite.de

Interessenkonflikt. Der korrespondierende Autor gibt an, dass kein Interessenkonflikt besteht.

\section{Literatur}

1. Beale R, Reinhart K, Brunkhorst FM et al (2009) Promoting global research excellence in severe sepsis (PROGRESS): lessons from an international sepsis registry. Infection 37:222-232

2. Teasdale G, Jennett $B$ (1974) Assessment of coma and impaired consciousness. A practical scale. Lancet 13:81-84

3. Ely EW, Inouye SK, Bernard GR et al (2001) Delirium in mechanically ventilated patients: validity and reliability of the confusion assessment method for the intensive care unit (CAM-ICU). JAMA 286:2703-2710

4. Luetz A, Heymann A, Radtke FM et al (2010) Different assessment tools for intensive care unit delirium: which score to use? Crit Care Med 38:409-418

5. Herridge MS, Tansey CM, Matté A et al (2011)

Functional disability 5 years after acute respiratory distress syndrome. N Engl J Med 364:1293-1304

6. Weber-Carstens S, Koch S, Spuler S et al (2009) Nonexcitable muscle membrane predicts intensive care unit-acquired paresis in mechanically ventilated, sedated patients. Crit Care Med 37:2632-2637

7. Schweickert WD, Pohlman MC, Pohlman AS et al (2009) Early physical and occupational therapy in mechanically ventilated, critically ill patients: a randomised controlled trial. Lancet 373:1874-1882

\section{Paul-Ehrlich- und Ludwig-Darm- staedter-Preis 2011 verliehen}

Der Chemiker und Biologe Prof. Cesare Montecucco aus Padua, Italien, erhält den mit 100.000 Euro dotierten Paul-Ehrlichund Ludwig-Darmstaedter-Preis 2011 für seine herausragenden Forschungsleistungen auf dem Gebiet pathogener Erkrankungen. Der Paul Ehrlich- und Ludwig Darmstaedter-Preis gehört zu den international renommiertesten Auszeichnungen, die in Deutschland auf dem Gebiet der Medizin vergeben werden.

Prof. Montecucco beschäftigte sich mit Erkrankungen, die durch pathogene Bakterien hervorgerufen werden. Montecucco untersuchte, wie das Tetanus-Neurotoxin synaptische Vesikel daran hindert Neurotransmitter freizusetzen.

Der mit insgesamt 60.000 Euro dotierte Paul-Ehrlich- und Ludwig-DarmstaedterNachwuchspreis 2011 geht an den Dresdner Biophysiker Dr. Stephan Grill vom MaxPlanck-Institut für molekulare Zellbiologie und Genetik und dem Max-Planck-Institut für Physik komplexer Systeme. Er wird für seine Beiträge auf dem Gebiet der Zellbiologie ausgezeichnet.

Dr. Grill hat eine Methode entwickelt, mit der mechanische Kräfte, die neben molekularen Mechanismen eine wesentliche Rolle bei der Zelldifferenzierung spielen, in lebenden Zellen gemessen werden können. Mit Hilfe eines Lasers kann er bestimmte Zellstrukturen minimal-invasiv zerstören. Deren Fragmente bewegen sich danach voneinander weg, wenn die Struktur unter mechanischer Spannung stand. Auf diese Weise erhält der Wissenschaftler einen Überblick darüber, wo in der Zelle mechanische Kräfte walten.

Quelle: Paul-Ehrlich-Stiftung, www.paul-ehrlich-stiftung.de 